\title{
Chirped pulse Raman amplification in plasma: high gain measurements
}

\author{
G. Vieux*, X. Yang, A. Lyachev† B. Ersfeld, J. Farmer, E. Brunetti, \\ M. Wiggins, R. Issac, G. Raj, D.A. Jaroszynski ${ }^{\ddagger}$ \\ University of Strathclyde, Department of Physics \\ Glasgow, G4 0NG, UK
}

\begin{abstract}
High power short pulse lasers are usually based on chirped pulse amplification (CPA), where a frequency chirped and temporarily stretched "seed" pulse is amplified by a broad-bandwidth solid state medium, which is usually pumped by a monochromatic "pump" laser. Here, we demonstrate the feasibility of using chirped pulse Raman amplification (CPRA) as a means of amplifying short pulses in plasma. In this scheme, a short seed pulse is amplified by a stretched and chirped pump pulse through Raman backscattering in a plasma channel. Unlike conventional CPA, each spectral component of the seed is amplified at different longitudinal positions determined by the resonance of the seed, pump and plasma wave, which excites a density echelon that acts as a "chirped" mirror and simultaneously backscatters and compresses the pump. Experimental evidence shows that it has potential as an ultra-broad bandwidth linear amplifier which dispenses with the need for large compressor gratings.
\end{abstract}

Keywords: laser-plasma interaction, Raman amplification, plasma channel

\section{INTRODUCTION}

High power ultra-short pulse lasers have become valuable tools for scientists exploring a wide range of phenomena and developing new technologies such as ultra-compact wakefield accelerators ${ }^{1}$ and compact light sources. ${ }^{2}$ Modern high power pulsed lasers rely on chirped pulse amplification (CPA), a technique originally developed in the 1980 's $\mathrm{s}^{3}$ to avoid damage to optical components. This technique involves amplifying a stretched, frequency chirped, "seed" pulse in a suitable solid state broadband amplifying medium excited by a monochromatic "pump" laser. After amplification, the seed pulse is compressed to ultra-short durations and high powers, currently up to several petawatts, ${ }^{4}$ in a compressor consisting of dispersive optical elements. Optical parametric chirped pulse amplification (OPCPA $)^{5}$ has been proposed as a way of both increasing the bandwidth and the power of high power amplifiers. However, the maximum output power of both CPA and OPCPA amplifiers is limited to several petawatts by the maximum intensities sustainable by optical components.

This limitation has led to the suggestion of using stimulated Raman back-scattering in plasma as an alternative parametric amplifying medium ${ }^{6}$ because plasma can sustain very high fields. Raman amplification occurs when two laser beams of slightly different frequencies collide in plasma to produce a beat wave with a frequency equal to the plasma frequency, $\omega_{p}=\sqrt{n e^{2} / \epsilon_{0} m}$, where $n$ is the plasma density, $e$ the electron charge, $\epsilon_{0}$ the permittivity of free space and $m$ the mass of an electron. The intrinsically narrow bandwidth of the Raman instability ${ }^{7}$ and its convective instability, ${ }^{8}$ due to the excitation of long lived plasma waves, limits the gain bandwidth achievable in the linear regime thus reducing its usefulness as a short pulse amplifier. Linear Raman amplification, ${ }^{7,9}$ which precedes the non-linear stage, is susceptible to undesirable noise amplification because of its intrinsically high gain. ${ }^{10,11}$ To avoid these limitations, Shvets et al. ${ }^{11-13}$ proposed taking advantage of the non-linearity of the medium at high intensities to produce soliton-like ultra-short pulses through pump-depletion or, at higher intensities, by operating in the Compton or superradiant regime where the plasma density echelon is dominated by the ponderomotive force associated with the beat wave of pump and seed. ${ }^{11,13}$ In this regime the gain becomes quadratic, with the intensity growing proportional with the square of the plasma density. Also

g.vieux@strath.ac.uk; phone: +44 141548 3103; fax: +44 1415522891

Dr. A. Lyachev is currently working at the Rutherford Appleton Laboratory, STFC, Chilton, UK

dino@phys.strath.ac.uk; phone: +44 141548 3057; fax: +44 1415522891 
the seed duration decreases as $\left|a_{1}\right|^{1 / 2}$, where $a_{1}$ is the normalised vector field potential amplitude of the seed pulse.

In this paper we present an experimental demonstration of efficient broadband chirped pulse Raman amplification obtained in the linear regime, which occurs when a long chirped pump pulse collides with a short counterpropagating seed pulse in a plasma column. The interaction acts as a distributed amplifier where different spectral components of the seed are amplified at different positions in the plasma through the creation of a "chirped" plasma density echelon. This behaves as a long chirped mirror which simultaneously backscatters and compresses the chirped pump pulse and effectively broadens the gain bandwidth to that of the pump. We show that the gain and the bandwidth of the amplifier depend on the plasma frequency and the chirp rate and spectral bandwidth of the pump. This contrasts with conventional CPA and OPCPA where the seed is chirped while the pump is usually monochromatic. The chirped pulse Raman amplifier (CPRA) has potential as a high fidelity ultra-short pulse high power linear amplifier or as a compressor of high energy chirped pulses from a conventional CPA amplifier, avoiding the requirement for extremely large and expensive optical elements and compressors in vast vacuum chambers. Furthermore, because CPRA is a three wave parametric interaction it provides a means of eliminating pre-pulses and pedestals which usually limit the usefulness of conventional solid state CPA amplifiers.

\section{THEORY}

\subsection{Stimulated Raman scattering}

Stimulated Raman scattering (SRS) occurs when an incident electromagnetic wave, with a frequency $\omega_{0}$ and wavenumber $\mathbf{k}_{\mathbf{0}}$, resonantly decays into a backscattered electromagnetic wave $\left(\omega_{1}, \mathbf{k}_{\mathbf{1}}\right)$ and a plasma wave $\left(\omega_{p}\right.$, $\mathbf{k}_{\mathbf{p}}$ ). It only occurs over a narrow frequency range when the resonance conditions $\omega_{0}=\omega_{1}+\omega_{p}$ and $\mathbf{k}_{\mathbf{0}}=\mathbf{k}_{\mathbf{1}}+\mathbf{k}_{\mathbf{p}}$ are approximately satisfied. ${ }^{12}$ In the linear SRS regime - at moderate seed and pump intensities $\left(\leq 10^{15} \mathrm{Wcm}^{-2}\right.$ for $800 \mathrm{~nm}$ radiation) - the amplitude of the seed grows exponentially as $a_{1} e^{\gamma t}$, where $\gamma$ is the growth rate and $t$ is the duration of the interaction. The gain coefficient, $\gamma$, is proportional to the pump field amplitude and the fourth root of the plasma density, i.e. $\gamma=a_{0}\left(\omega_{0} \omega_{p} / 2\right)^{1 / 2},{ }^{7,9}$ for circularly polarised monochromatic beams, where the normalised vector potential is given by $a_{i}=e A_{i} / m c$ and $A_{i}$ is the vector potential amplitude and $c$ the speed of light. For low seed intensities, the energy transfer is small and pump depletion can be neglected i.e. $a_{0} \simeq$ const. Despite the significant available gain, amplification can be diminished by several concurrent phenomena such as spontaneous Raman backscattering leading to early pump depletion, Raman forward scattering, temperature increase due to inverse bremsstrahlung heating, Landau damping, particle trapping etc.. ${ }^{14}$ Furthermore, the narrow gain bandwidth, equal to $2 \gamma$, in the linear SRS regime and a convective instability lead to temporal stretching of the seed pulse and a group velocity equal to half the speed of light. ${ }^{8,12}$ The nonlinear SRS $^{11,13}$ regime begins when pump depletion becomes noticeable and depression of gain at the rear of the pulse leads to the formation of a self-similar " $\pi$ " pulse growing linearly in amplitude and quadratically in intensity, $I \propto t^{2}$, while its temporal width $\tau$ shrinks as $\propto 1 / \tau$, leading to pulse compression. Ultimately, when $a \geq a_{w b}=\left(\omega_{p} / 4 \omega_{0}\right)^{3 / 2}$ (for monochromatic waves), the density modulation approaches $100 \%$ and the plasma wave breaks. ${ }^{13}$ The SRS instability can also evolve into the Compton regime when the ponderomotive force exceeds that exerted by the plasma wave i.e. the bounce frequency, $\omega_{B}=2 \omega_{0} \sqrt{a_{0} a_{1}}$, of plasma electrons in the ponderomotive well exceeds the plasma frequency, $\omega_{B} \geq \omega_{p} .{ }^{11}$ Again, a self-similar pulse evolves with an intensity increasing quadratically with propagation distance, a scaling that is characteristic of superradiance. ${ }^{15,16}$

\subsection{Chirped pulse Raman amplification}

To avoid the disadvantages of using a monochromatic pump pulse as discussed above, we show in the following that a chirped pump pulse allows high gain to be achieved at moderate efficiencies. An important observation of our work is that the chirp of the pump restricts the effective interaction length. For monochromatic pump and seed, the gain coefficient depends on the detuning $\Delta=\omega_{0}-\omega_{1}-\omega_{p}: \gamma(\Delta)=\sqrt{\gamma_{0}^{2}-\Delta^{2} / 4}$. In the case of a pump pulse with its frequency chirped at a rate $\alpha$, such that $\omega_{0}=\omega_{0}^{(0)}+\alpha(t+z / c)$, the chirp determines the longitudinal position at which each frequency component of the seed will be amplified. This is because the local detuning, $\delta(z, t) \sim \alpha(t+z / c)$, allows the plasma wave to grow only while $|\delta(z, t)| \leq 2 \gamma_{0}$ i.e. while the component remains within the resonance bandwidth for that spectral component. This has the effect of distributing the gain both in 
frequency and position, resulting in a broad bandwidth amplifier with a gain independent of the plasma channel length, as shown schematically in Fig. 1. Gain for each spectral component is restricted to a time $\tau \sim \pi\left|\gamma_{0} / 2 \alpha\right|$ by dephasing and detuning. The plasma wave amplitude also saturates at $\delta n / n_{0} \sim s_{\omega_{S}} \sqrt{\alpha / \pi} e^{\pi \gamma_{0}^{2} / 2 \alpha}$, where $s_{\omega_{S}(z)}$ is the Fourier component of the seed at the Stokes frequency corresponding to the position $z,{ }^{17}$ which allows wavebreaking to be avoided. In other words, for a seed with a normalised bandwidth $\Delta_{s}$, wavebreaking is governed by the field amplitude of the Fourier component $s_{\omega}=a_{1} / \Delta_{s}$ driving the plasma wave and not the total field amplitude as would be the case for monochromatic waves. Wavebreaking is avoided as long as $\left|s_{\omega}\right| \leq \sqrt{\alpha / \pi} e^{\pi \gamma_{0}^{2} / 2 \alpha}$, which increases the threshold at which wavebreaking occurs by more than an order of magnitude. ${ }^{17}$

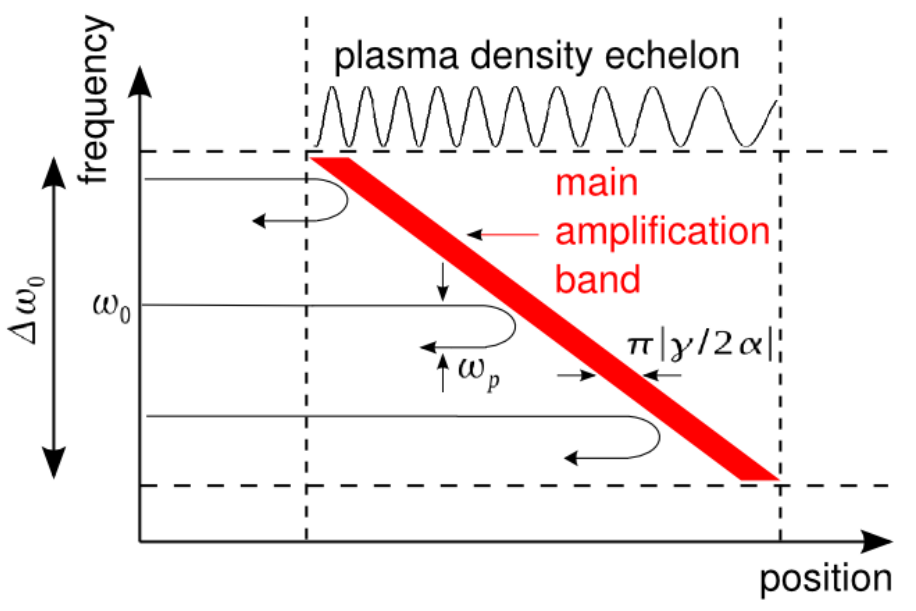

Figure 1. Schematic of a chirped plasma grating.

The fields scattered off the plasma wave superpose coherently just behind the seed, but dephase with increasing distance from it. Consequently, both seed amplitude and spectral bandwidth grow proportional to the propagation distance, which contrasts with the continual stretching of the seed profile expected for the case of a monochromatic pump. The scattered fields effectively carry out a Fourier transform of the seed "on the fly" which leads to self-similar growth that exhibits superradiant scaling usually associated with the nonlinear Raman and Compton regimes. ${ }^{17}$ The evolution of the seed bandwidth is limited by the overlap of the bandwidths of seed and pump, and has a maximum equal to $\omega_{p}$. Since saturation of the growth of the plasma wave depends on the pump intensity rather than its amplitude (as would be expected for a monochromatic pump), the same applies to the overall gain bandwidth. It should be noted that a similar dependence on chirp will also occur if we chirp the plasma density. ${ }^{13}$

Such schemes could be of great interest for the future development of a CPA Raman amplifier. Because the gain bandwidth in the linear regime is given by the spectral bandwidth of the pump beam it provides an alternative to $\mathrm{OPCPA}^{5}$ for the generation of ultra-short, ultra-intense laser pulses with the advantage that the power is not limited by breakdown of the amplifying medium. It should also be emphasised that using a monochromatic pump and a plasma with a density gradient leads to the same result.

\section{EXPERIMENTAL SET-UP}

Several groups have investigated Raman amplification in plasma experimentally using short micro-capillaries ${ }^{18}$ or gas jets ${ }^{10} \leq 2 \mathrm{~mm}$ long. For some time the main results obtained were an amplification by a factor 8 demonstrated for a plasma density of $3 \times 10^{20} \mathrm{~cm}^{-3}$. Ping et al. ${ }^{18,19}$ observed gains of more than three orders of magnitude. However, they observed gain bandwidths, $\delta \lambda / \lambda$, less than $0.3 \%$ in the linear regime. Also, previous experiments have shown evidence of non-linear amplification ${ }^{19}$ and synchrotron oscillations indicating Compton amplification ${ }^{20}$ and at saturation a bandwidth increases to $2 \% .^{21}$ However, these experiments showed 
a spiky spectra and low efficiency. Recently, an energy gain of 350 times was observed in a double-pass Raman backscattering amplifier in a $2 \mathrm{~mm}$ long gas jet. ${ }^{22,23}$ In these studies, the seed energy increased from $16 \mu \mathrm{J}$ to $5.6 \mathrm{~mJ}$ and pulse compression was observed, with the initial seed duration of 500 fs decreasing down to 50 fs. The energy transfer efficiency of this amplifier was $6.4 \%$. While this is still far from the pump depletion regime, these latest results are very promising.

In contrast with previous experiments, we have studied efficient broad-band chirped pulse Raman amplification in the linear regime and demonstrated its viability as a high fidelity linear broad bandwidth amplifier. To achieve this a co-linear counter-propagating beam experiment in a plasma channel was set-up using the TOPS high power laser system as is schematically shown in Fig.(2). ${ }^{24}$ The laser system, based on the CPA technique, provides, before the final amplifier, a $350 \mathrm{~mJ}, \approx 200 \mathrm{ps}$ chirped beam centered at $\lambda_{0}=800 \mathrm{~nm}$ at a $10 \mathrm{~Hz}$ repetition rate. A beam splitter splits the beam into a seed and a pump which focus on either side of a $4 \mathrm{~cm} \mathrm{long,} 300 \mu \mathrm{m}$ internal diameter plasma channel waveguide using F/40 spherical mirrors. The pump, containing $90 \%$ of the initial beam energy, propagates through a delay line before being focused into the capillary to allow perfect synchronization with the seed. Prior to the interaction, the seed beam is compressed down to a minimum duration of $50 \mathrm{fs}$ and its spectrum down-shifted by self-phase modulation and filamentation in a $1 \mathrm{~m}$ long argon tube.

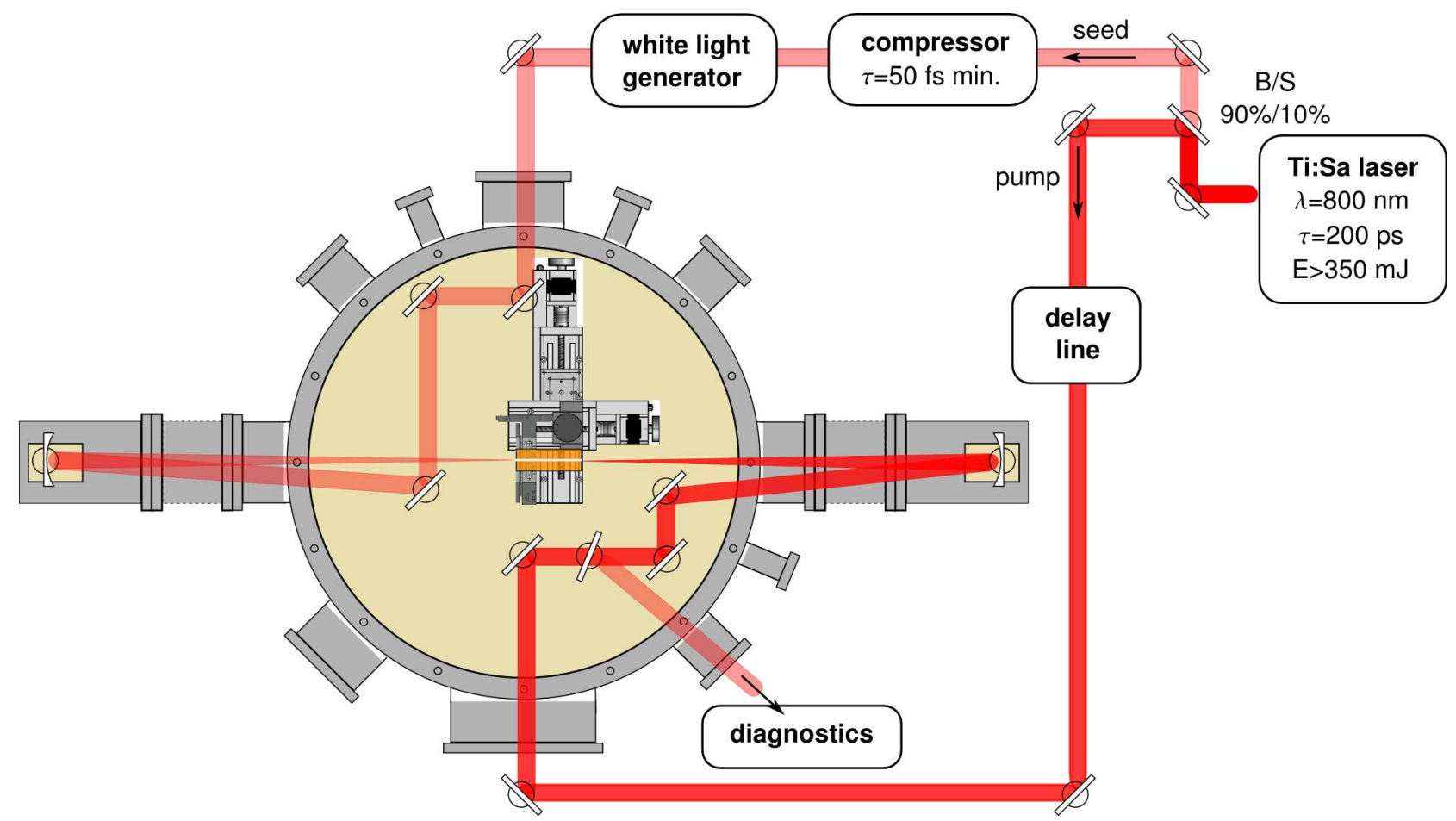

Figure 2. Schematic of the experimental set-up.

This allows a perfect match between the frequency difference of the two beams and the plasma frequency. However, it should be noted that the bandwidths of pump and seed are sufficiently large $(\delta \lambda=33 \mathrm{~nm}$ maximum $)$ for the beat wave of their respective frequency components to excite a Langmuir wave, which leads to SRS, even when pump and pulse spectra are identical. The seed energy, at the entrance of the chamber can be varied from $250 \mu \mathrm{J}$ to $12 \mathrm{~mJ}$. However, spectral broadening due to self-phase modulation is not possible at higher energies.

The plasma waveguide, mounted on a 5-axis stage, is formed by a high voltage discharge in a $\mathrm{H}_{2}$ filled capillary. ${ }^{25,26}$ The plasma cools against the capillary wall to form a parabolic radial density distribution, $N(r)=$ $N(0)+\Delta N\left(r / r_{m}\right)^{2},{ }^{27}$ with an on-axis density $\mathrm{N}(0) \approx 10^{18} \mathrm{~cm}^{-3}$, and $\Delta N$ is the increase to the boundary wall at $r_{m}$. This gives a value of $\omega_{p} / \omega_{0} \approx 2 \%$. The laser beam couples to the fundamental mode of the waveguide provided the spot size is $w_{M}=\left(r_{m} /\left(\pi r_{e} \Delta N\right)\right)^{1 / 4}$, where $r_{e}$ is the classical electron radius. 
After interaction, $4 \%$ of the seed is split off to two diagnostic systems: an imaging system consisting of a 12 bit CCD camera with a $\times 4$ microscope objective for imaging the beam input and output, and a spectrometer for measuring the gain as a function of wavelength. Fig.(3) illustrates the guiding properties of a $4 \mathrm{~cm}$ long waveguide, as recorded on the imaging system, when the matching condition is perfectly satisfied. The pump beam, of duration $200 \mathrm{ps}$, energy $180 \mathrm{~mJ}$, and waist $43 \mu \mathrm{m}$, propagates through the plasma channel with an energy transmission $>90 \%$ on average. The output beam has the same waist size and maintains a Gaussian profile. Furthermore, no ionisation-induced blue shifting of the spectrum is observed indicating that the plasma is fully ionized.

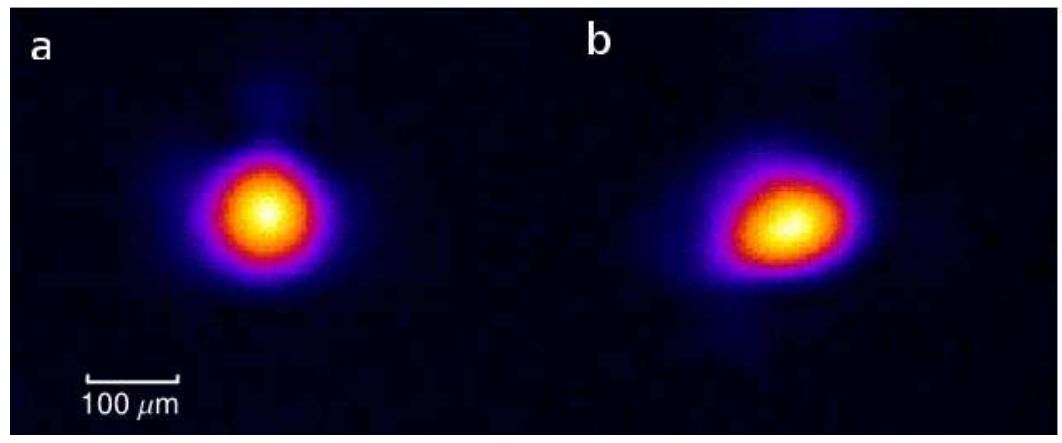

Figure 3. Transmission example through a $4 \mathrm{~cm}$ long plasma channel; a) input beam, b) output beam.

\section{EXPERIMENTAL RESULTS}

In the first experiment, a $18.5 \mathrm{~nm}$ bandwidth, $800 \mathrm{~nm}$ wavelength, $3 \mathrm{~mJ}$, seed pulse, with $a_{1} \approx 3.2 \times 10^{-3}$, collides with a $170 \mathrm{ps}, 220 \mathrm{~mJ}$, pump pulse, with $a_{0} \approx 2.4 \times 10^{-3}$, chirped at a rate $\alpha=\Delta \omega_{0} / \Delta T \approx 3.13 \times 10^{23}$ $\mathrm{s}^{-2}$, in a $40 \mathrm{~mm}$ long preformed plasma waveguide channel. Here, the central wavelength of the seed pulse is not detuned from that of the pump, thus energy can be exchanged either way between pump and seed because of their large respective spectral bandwidths. Amplification of the seed signal is observed at the Stokes wavelength, while a loss is observed at the anti-Stokes wavelength, which can be controlled by varying the relative time delays between seed and pump. The seed pulse is slightly streched through the compressor to a duration of 610 fs to avoid white light generation in optical elements. The product $\left|a_{0} a_{1}\right|$ is a factor 12 below the threshold for entering the Compton regime. The large gain coefficient $\gamma=5.65 \times 10^{11} \mathrm{rads}^{-1}$ would lead to the nonlinear stage in just $1.3 \mathrm{~mm}$ for a monochromatic pump. However, the large chirp reduces the length over which the gain occurs to $c \tau \approx 0.5 \mathrm{~mm}$, thus dramatically reducing the gain. Using the exprimental parameters the predicted growth of each spectral component where resonance is achievable is $e^{\pi \gamma^{2} / 2 \alpha} \sim 2$, which compares well with the maximum measured intensity gain of $\approx 250 \%$ for carefully optimised pump laser as shown in Fig.(4). For these gain measurements the seed energy was increased to $12 \mathrm{~mJ}$. The efficiency of the amplifier is estimated to be $5 \%$ for the segment of the pump pulse resonant with the seed. The spectral bandwidth of the measured gain is $\Delta \lambda_{G} \approx 11 \mathrm{~nm}$, which is approximatively $60 \%$ of the pump bandwidth. This is consistent with only half of the seed spectrum in resonance with the pump spectrum.

The efficiency $\eta(\lambda)$ is determined as a function of the wavelength by calculating the energy transferred from the pump to the seed, taking into account the difference in frequency $\left(\omega_{p} / \omega_{0} \approx 2 \%\right)$ imposed by the resonant condition. The gain curve $\mathrm{G}(\lambda)$ is measured by recording the seed spectra with and without the pump, $I^{\prime}(\lambda)$ and $I(\lambda)$ respectively, which enables $e^{2 \gamma t}-1$ to be evaluated from $G(\lambda)=\left(I^{\prime}(\lambda)-I(\lambda)\right) / I(\lambda)$.

In a second experiment, a cut-off filter was used in place of the beam splitter to red-shift the seed spectrum with respect to the pump spectrum. The higher frequencies of the initial laser pulse are reflected by the cut-off filter to form the pump while the lower frequency part is used as the seed. Fig.(5) shows a typical pump and initial seed spectrum as well as the amplified seed spectrum and corresponding gain function.

This result is obtained for a $80 \mathrm{fs}, 3 \mathrm{~mJ}$ seed interacting with a $220 \mathrm{~mJ}$ pump beam in a plasma channel with a density estimated to be $1.3 \times 10^{18} \mathrm{~cm}^{-3}$. A peak gain of $900 \%$ is observed leading to an energy gain of $32 \%$. 


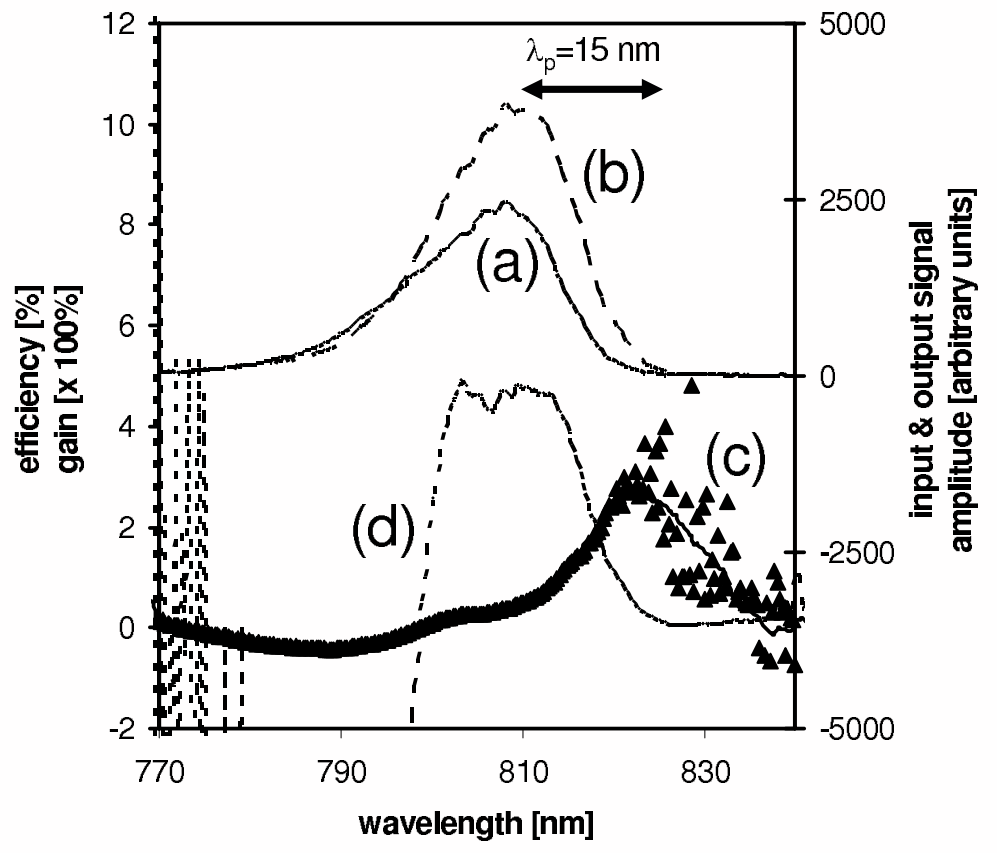

Figure 4. (a) Seed spectra without pump, (b) with pump, (c) gain $\mathrm{G}(\lambda)(\times 100 \%)$ and (d) efficiency $\eta(\lambda)(\times 1 \%)$, for $a_{0}=2.54 \times 10^{-3}$.

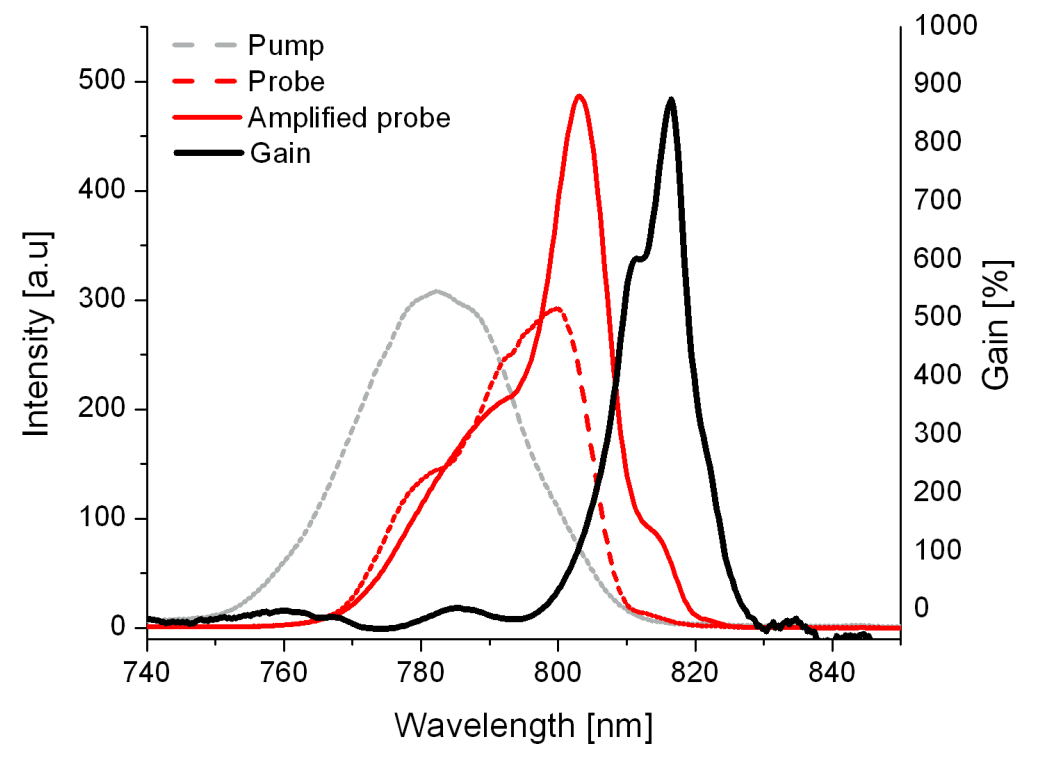

Figure 5. Typical gain measurement. 
The energy transfer efficiency is of the order of $3.5 \%$ for the amplified region of the spectrum in this case. It can clearly be seen that the energy gain is limited because the seed spectrum is not sufficiently down-shifted and thus the interaction is limited to only a part of the pump spectrum.

The dependence of the maximum peak gain as a function of plasma density is presented in Fig.(6), together with theoretical predictions.

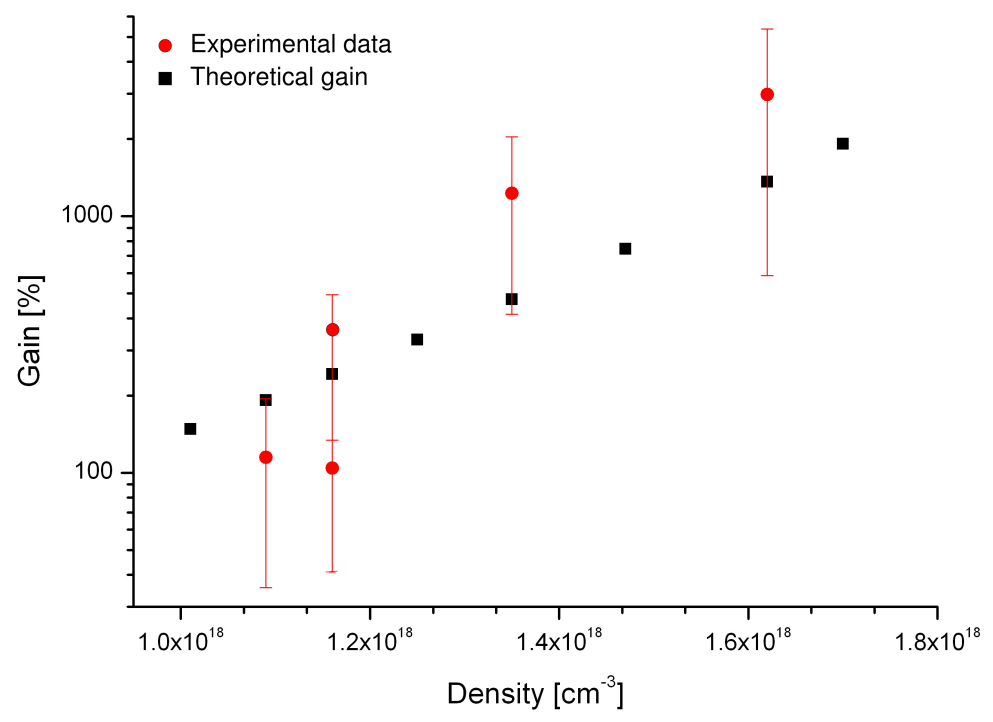

Figure 6. Gain measurements as a function of plasma density.

The density is increased from $10^{18} \mathrm{~cm}^{-3}$ to $1.6 \times 10^{18} \mathrm{~cm}^{-3}$, leading to the peak gain increasing from $150 \%$ to $3000 \%$. The numerical predictions, taking into account the chirp of the pump, show good agreement with the measurements.

Finally, the seed duration has been measured, using a single-shot autocorrelator, after amplification and compared with its initial duration. Fig.(7) presents the relative changes measured for different initial seed durations ranging from 80 fs to $1.2 \mathrm{ps}$.

For durations below $500 \mathrm{fs}$, the seed appears to be stretched, with a relative duration increasing as the initial pulse duration decreases. For shorter seed beams with a duration measured to be $\sim 80 \mathrm{fs}$, the pulse duration increases by $40 \%$. However, for durations above $500 \mathrm{fs}$, the seed pulse is compressed by a constant compression factor of $\sim 10 \%$. The interpretation of this result is that interaction takes place with a chirped seed pulse over only a part of its spectrum. Because the frequencies are spatially distributed, only a part of the spectrum is amplified leading to an effective compression of the seed pulse as is illustrated in Fig.(8).

\section{CONCLUSION}

We have demonstrated that chirped pulse Raman amplification could be used to develop an efficient laser pulse amplifier with the potential of reaching the nonlinear or Compton regime. While the gain for each seed frequency is limited to $e^{\pi \gamma_{0}^{2} / 2 \alpha}$, we still observe an energy gain of $32 \%$ when colliding a $80 \mathrm{fs}, 3 \mathrm{~mJ}$ seed with a $170 \mathrm{ps}, 220$ $\mathrm{mJ}$ pump in $4 \mathrm{~cm}$ long plasma channel with an electron density of $1.3 \times 10^{18} \mathrm{~cm}^{-3}$. The energy transfer efficiency is of the order of $3.5-5 \%$ over a bandwidth given by the pump bandwidth. Change in pulse duration was also studied. While the seed duration increases by up to $40 \%$ for bandwidth-limited or close to bandwidth-limited pulses, for stretched seeds a compression factor of $10 \%$ is measured. This observation is not related to non-linear broadening of the spectrum bandwidth, which should occur at high intensities. In our case, this is expained by amplification of only part of the spectral frequencies with are spatially distributed due to the chirp. 


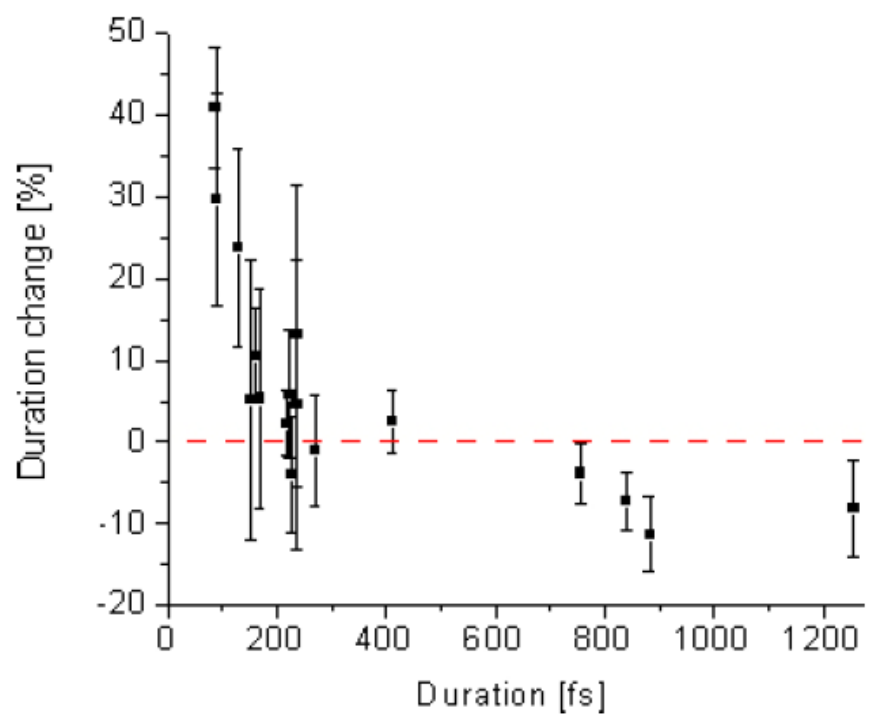

Figure 7. Pulse duration change as a function of initial pulse duration.
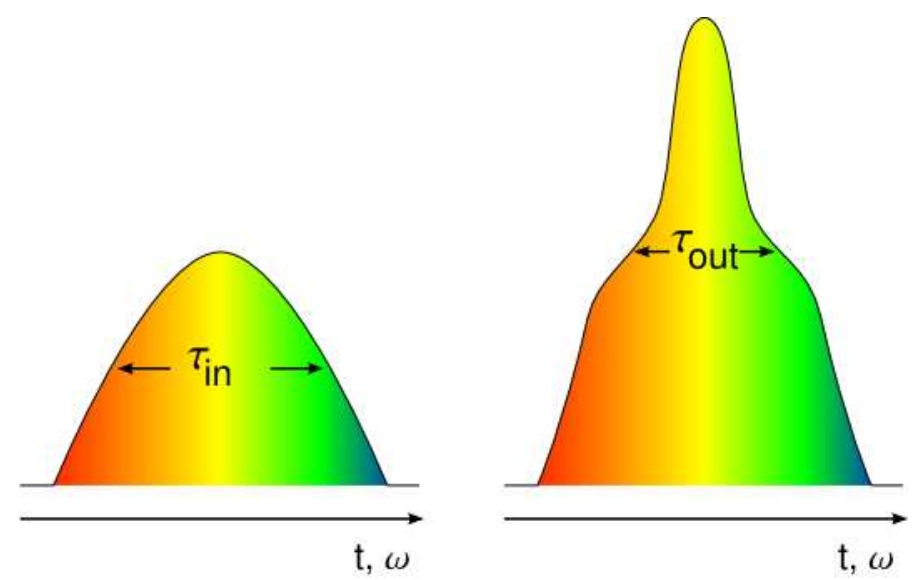

Figure 8. Schematic of effective pulse compression due to Raman narrow gain bandwidth. 
To study high efficiency amplification, self-phase modulation through filamentation in an Argon tube is being developed. This enables the control of the frequency shift of the seed while conserving the pulse bandwidth while maintening a short pulse. Experiments are currently in progress.

\section{REFERENCES}

1. T. Tajima and T. Taniuti, "Nonlinear interaction of photons and phonons in electron-positron plasmas," Phys. Rev. A 42(6), p. 3587, 1990.

2. D. A. Jaroszynski and G. Vieux, "Coherent radiation sources based on laser plasma accelerators," AIP Conf. Proc. 647, p. 902, 2002.

3. D. Strickland and G. Mourou, "Compression of amplified chirped optical pulses," Opt. comm. 56, p. 219, 1985.

4. C. B. Edwards and et al, "Vulcan petawatt interaction facility," Proc. SPIE Int. Soc. Opt. Eng. 4948, p. $444,2003$.

5. I. N. Ross, P. Matousek, M. Towrie, A. J. Langley, and J. L. Collier, "The prospects for ultrashort pulse duration and ultrahigh intensity using optical parametric chirped pulse amplifiers," Opt. Comm. 144, p. 125, 1997.

6. G. Shvets, N. J. Fisch, A. Pukhov, and J. Meyer ter Vehn, "Superradiant amplification of an ultrashort laser pulse in a plasma by a counterpropagating pump," Phys. Rev. Lett. 81(22), p. 4879, 1998.

7. W. Kruer, The Physics of Laser Plasma Interaction, Addison-Wesley, Reading, MA, 1988.

8. D. L. Bobroff and H. A. Haus, "Impulse response of active coupled wave systems," J. Appl. Phys. 38, p. 390, 1967.

9. D. W. Forslund, J. M. Kindel, and E. L. Lindman, "Theory of stimulated scattering processes in laserirradiated plasmas," Phys. Fluids 18(8), p. 1002, 1975.

10. Y. Ping, I. Geltner, and S. Suckewer, "Raman backscattering and amplification in a gas jet plasma," Phys. Rev. E 67, p. 016401, 2003.

11. G. Shvets, J. S. Wurtele, and B. A. Shadwick, "Analysis and simulation of raman backscatter in underdense plasmas," Phys. Plasmas 4(5), p. 1872, 1997.

12. V. M. Malkin, G. Shvets, and N. J. Fisch, "Ultra-powerful compact amplifiers for short laser pulses," Phys. Plasmas 7(5), p. 2232, 2000.

13. V. M. Malkin, G. Shvets, and N. J. Fisch, "Fast compression of laser beams to highly overcritical powers," Phys. Rev. Lett. 82(22), p. 4448, 1999.

14. L. R. Berger, D. S. Clark, A. A. Sodolov, E. J. Valeo, and N. J. Fisch, "Inverse bremsstrahlung stabilization of noise in the generation of ultrashort intense pulses by backward raman amplification," Phys. Plasmas 11, p. 1931, 2004.

15. R. H. Dicke, "Coherence in spontaneous radiation processes," Phys. Rev. 93, p. 99, 1954.

16. D. A. Jaroszynski, P. Chaix, and N. Piovella, "Superradiance in a short-pulse free-electron-laser oscillator," Phys. Rev. Lett. 78(9), p. 1699, 1997.

17. B. Ersfeld and D. A. Jaroszynski, "Superradiant linear raman amplification in plasma using a chirped pump pulse," Phys. Rev. Lett. 95, p. 165002, 2005.

18. Y. Ping, I. Geltner, A. Morozov, N. J. Fisch, and S. Suckewer, "Raman amplification of ultrashort laser pulses in microcapillary plasmas," Phys. Rev. E 66, p. 046401, 2002.

19. W. Cheng, Y. Avitzour, Y. Ping, S. Suckewer, N. Fisch, M. Hur, and J. Wurtele, "Reaching the nonlinear regime of raman amplification of ultrashort laser pulses," Phys. Rev. Lett. 94, p. 045033, 2005.

20. M. Dreher, E. Takahashi, J. Meyer-ter-Vehn, and K.-J. Witte, "Observation of superradiant amplification of ultrashort laser pulses in a plasma," Phys. Rev. Lett. 93, p. 095001, 2004.

21. A. A. Balakin, D. V. Kartashov, A. M. Kiselev, S. A. Skobelev, A. N. Stepanov, and G. M. Fraiman, "Laser pulse amplification upon raman backscattering in plasma produced in dielectric capillaries," Jetp. Lett. 80, p. $12,2004$.

22. J. Ren, W. Cheng, S. Li, and S. Suckewer, "A new method for generating ultraintense and ultrashort laser pulses," Nat. Phys. 3, p. 732, 2007. 
23. J. Ren, S. Li, A. Morozov, S. Suckewer, N. A. Yampolsky, and V. M. Malkin, "A compact double-pass raman backscattering amplifier/compressor," Phys. Plasmas 15, p. 056702, 2008.

24. D. A. Jaroszynski, B. Ersfeld, G. Giraud, S. Jamison, D. R. Jones, R. C. Issac, B. M. W. McNeil, A. D. R. Phelps, G. R. M. Robb, H. Sandison, G. Vieux, S. M. Wiggins, and K. Wynne, "The strathclyde terahertz to optical pulse source (tops)," Nucl. Inst. and Meth. in Phys. Res. Sect. A - Acc. Spect. Det. and Assoc. Equip. 445, p. 317, 2000.

25. D. J. Spence and S. M. Hooker, "Investigation of a hydrogen plasma waveguide," Phys. Rev. E 63, p. 015401, 2001.

26. D. A. Jaroszynski, R. Bingham, E. Brunetti, B. Ersfeld, J. Gallacher, B. van der Geer, R. Issac, S. P. Jamison, D. Jones, M. de Loos, A. Lyachev, V. Pavlov, A. Reitsma, Y. Saveliev, G. Vieux, and S. M. Wiggins, "Radiation sources based on laser-plasma interactions," Phil. Trans. R. Soc. A 364, p. 689, 2006.

27. D. J. Spence, P. D. S. Burnett, and S. M. Hooker, "Measurement of the electron-density profile in a discharge-ablated capillary waveguide," Opt. Lett. 24(14), p. 993, 1999. 